\title{
Simulation of heat transfer in intricately- configured polymer composite structures of instrumented container type
}

\author{
Mikhail N. Slitkov ${ }^{1}$, Alexander A. Kulkov ${ }^{1}$, and Anatoly N. Garashchenko, \\ ${ }^{1}$ Central Research Institute for Special Machinery, Moscow, Russia
}

\begin{abstract}
Method of mathematical simulation of heat transfer processes in polymer composite (PC) products with intricate configuration, being an alternative of using up-to-date commercial software complexes has been developed. On the example of PC container with instrumentation and fiberglass electric heaters located in it, a mathematical model describing unsteady temperature field (a system of nonlinear differential heat balance equations for each element) has been formulated. Features of heat transfer between elements (heaters, instrumentation, enclosing structures) were taken into account. The verification of the method was conducted by comparing of theoretical temperature distributions with results of measurements in experiments with simplified variant of the structure. The developed method is effective, in particular, for such PC products as containers, modules, bunkers and vessels. It allows us to specify optimum operation modes for heating elements, operational parameters for conditioners and funs, heat insulation characteristics for providing a given level of air temperature inside objects in winter and summer service periods.
\end{abstract}

\section{Introduction}

Containers, modules, bunkers and vessels that should be provided with a given heat mode, are the advanced kind of products of polymer composite materials. PC containers (assemblies) for locating instrumentation which should function under different climatic conditions are such typical products. For maintaining a given temperature mode in these assemblies, it is efficiently to use thin-layered fiberglass electric heaters (NEST) that have been widely practiced (from household application to maintaining a given temperature mode in elements of the rocket and space engineering) [1,2].

To design such assemblies, the information about time history of temperature of all comprised elements is necessary. It is also necessary to control given heating modes. The listed PC products have, as a rule, an intricate configuration that substantially hampers the simulation of temperature fields in structures. The use of up-to-date commercial software complexes, in particular [3, 4], is impossible for a whole number of objective reasons. In such complexes, for example, not all possible variants of heat transfer between modeled

\footnotetext{
${ }^{*}$ Corresponding author: a.n.gar@mail.ru
} 
objects and environment are provided for, or it is taken into account in the simplified form. The correction of commercial software packages is not possible.

\section{Statement of the problem}

It is necessary to use alternative methods of computer simulation. As such method, this paper proposes a method providing the realization of any variants of complex physical models of heat transfer processes in products. A mathematical model describing unsteady temperature field of an assembly, in particular, a PC container is based on a system of usual differential nonlinear heat balance equations for each separate assembly element. The increase of the number of such elements causes only appropriate increase of the number of equations. Let us consider the mathematical model, a problem solution method and obtaining of calculation results with its assistance on the example of a container for electronic equipment. Its schematic is presented in Fig. 1.
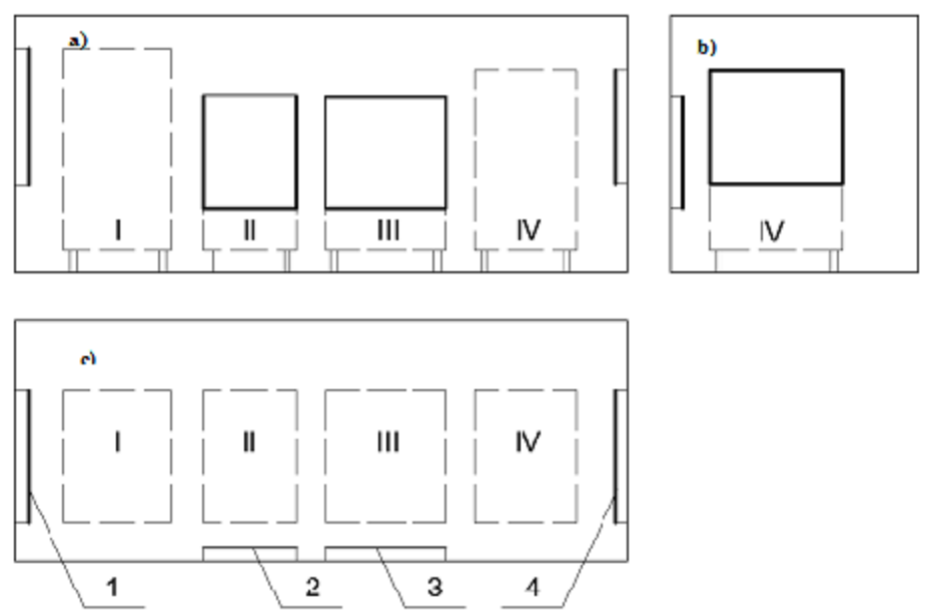

Fig. 1. Problem solution domain (inner volume of container: a) back side; b) right side; c) top view).

A box-shaped container $2000 \mathrm{~mm}$ in length, $700 \mathrm{~mm}$ in width and $1000 \mathrm{~mm}$ in height was adopted for the simulation. The front wall has two doors for access to the container. Four blocks of electronic equipment (I, II, III, IV) and four NESTs $(1,2,3,4)$ are located inside the container. NESTs are installed in that way that each of them radiates a heat only on a device standing opposite it and on a surface section of a wall located behind NEST. Outer and inner layers of vertical walls, a cover and a bottom are made of glass fiber reinforced plastic. The intermediate layer of walls, a bottom and covers is made of polyurethane foam (heat-insulating layer). Blocks are set on supports with a gap from floor equal to $20 \mathrm{~mm}$. Distances from blocks I and IV to lateral walls are $150 \mathrm{~mm}$ and $100 \mathrm{~mm}$ respectively. The distance between blocks is $80 \mathrm{~mm}$, and between blocks and longitudinal walls is $170 \mathrm{~mm}$ (see Fig. 1).

It is adopted that the heat in the container in winter period of time is evolved by all four NESTs as well as two instrumentation blocks (I and II). The mode of maintaining the air temperature over the range of $+10{ }^{\circ} \mathrm{C} \ldots+25{ }^{\circ} \mathrm{C}$ inside the container when operating in winter and summer periods at the ambient temperature from $-50{ }^{\circ} \mathrm{C}$ to $+40{ }^{\circ} \mathrm{C}$ was given. In summer period, solar radiation fluxes were taken into account, and an air-conditioner and a fun were used to maintain the given level of the air temperature inside the container. In winter period, heaters were switched out when reaching the upper boundary of the indicated range and switched on when reaching the bottom boundary. 
To determine the temperature field of an assembly "container-blocks-NEST", including the mutual heat interaction, it was broken into 22 elements. For each element, a differential heat balance equation was formed. Since heat bonds between elements include temperatures to be determined, the equations were nonlinear and a system of equations was solved by one of numerical integration methods.

The admissibility of such approach has been validated by comparing data of model experiment with calculation results. A model container with relatively simple structure (as compared with an assembly in Fig. 1) was used. Temperature of basic elements of the container was measured. The experiments were conducted in two stages (the first - without thermal control, the second - with thermal control). Dimensions of the model container are $460 \mathrm{~mm} \times 420 \mathrm{~mm}$, height is $390 \mathrm{~mm}$. Dimensions of the instrumentation block are 140 $\mathrm{mm} \times 220 \mathrm{~mm}$, height and weight are $100 \mathrm{~mm}$ and $4.5 \mathrm{~kg}$ respectively. The walls, cover and bottom are multilayered and made of packing board (inner layer), acrylic plastic (intermediate layer) and molding material (outer layer). The thickness of walls is $6 \mathrm{~mm}$, of a cover is $12 \mathrm{~mm}$, of a bottom is $42.5 \mathrm{~mm}$. The gap between NEST and a wall is $20 \mathrm{~mm}$, between a block and a wall is $35 \mathrm{~mm}$, between a block and floor is $10 \mathrm{~mm}$. Positioning elements in the container are shown in Fig. 2. Thermocouples inside the container were installed in the following way: three on NEST; two on a block (one on the surface, other inside); two in air at different heights; one behind NEST; one by one on bottom and vertical walls. Results of the experiments are presented in Fig. 3.

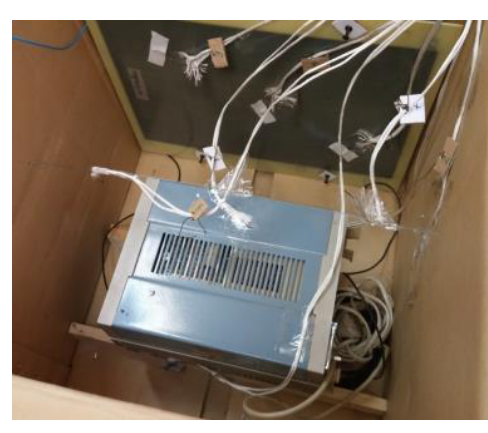

Fig. 2. General view of model container.

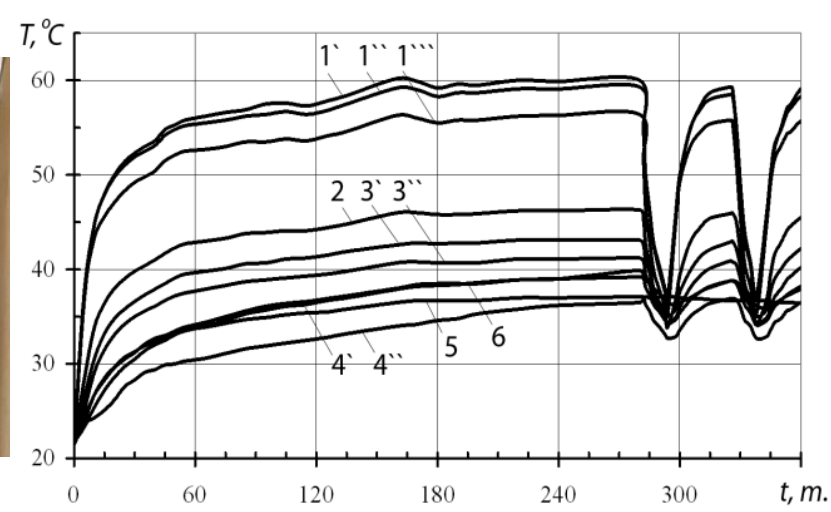

Fig. 3. Changing of element temperature in experiments with thermal control: 1', 1', 1',' - NEST; 2 - inner wall surface in NEST zone; 3', 3' ' - air; 4' - surface of block and its inner part (4'); 5 - surface of bottom and wall (6) outside NEST.

For the model container under consideration, a system of 8 differential heat balance equations was formed: 1 - NEST, 2 - wall in NEST zone, 3 - air, 4 - instrumentation block, 5 - floor in block zone, 6 - floor outside block zone, 7 - vertical walls outside NEST zone, 8 - cover). The equation stated for NEST is presented as an example:

$$
C_{1} \frac{d T_{1}}{d t}=P_{1}-K_{1-2}\left(T_{1}-T_{2}\right)-K_{1-3}\left(T_{1}-T_{3}\right)-K_{1-4}\left(T_{1}-T_{4}\right)-K_{1-4}\left(T_{1}-T_{4}\right)-K_{1-4}\left(T_{1}-T_{4}\right),
$$

where $P_{1}$ is NEST power;

$K_{1-2}$ is parameter of heat transfer by effective heat conduction from heater to wall, taking into account re-emission of surfaces and natural convection in plane slit [3]; $K_{1-3}$ is parameter of heat transfer by natural convection from heater to air; 
$K_{1-4}, K_{1-5}, K_{1-8}$ are parameters of heat transfer by radiation from heater to elements whose angle coefficients were determined with due account of positioning mutually irradiating surfaces [3].

In this statement of a problem, the temperature of NEST was supposed equal for the whole its surface. In reality, the distinction in temperatures of its upper and bottom parts can reach tens degrees at the expense of non-uniform heat removal because of natural convection (see Fig. 3). For calculating the temperature distribution in vertical direction, NEST was broken into $n$ sections and the heat balance equation was formed for each of them. This problem should be solved separately when using already calculated temperaturetime characteristics of all elements and including a dependence of natural-convective heat transfer coefficient on vertical coordinate of a heater.

\section{Results and discussion}

The system of differential nonlinear heat balance equations was solved by the 4th-order Runge-Kutt method. When calculating it was given that NEST power to be evolved under thermal control is $40 \mathrm{~W}$ (without thermal control is $30 \mathrm{~W}$ ); specific heat capacity of instrumentation block is $850 \mathrm{~J} / \mathrm{kg} \cdot \mathrm{K}$; thermal control limits are $+35^{\circ} \mathrm{C} \ldots+43{ }^{\circ} \mathrm{C}$. The results of calculating temperatures of model container elements are presented in Fig. 4. Their comparison with the results of experiments (see, in particular, Fig. 3) showed a good correspondence that witnesses the validity of the stated calculation method.

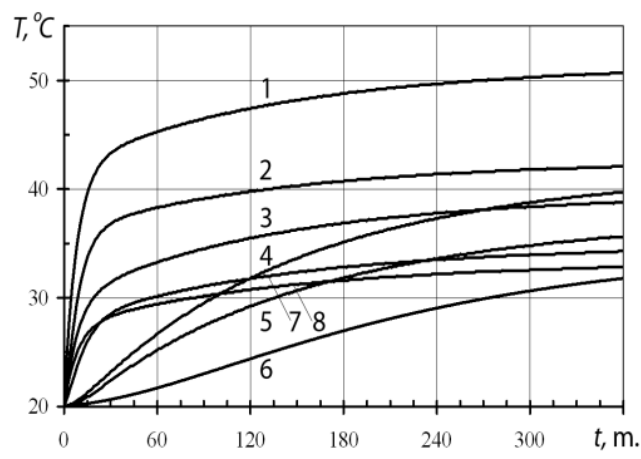

a)

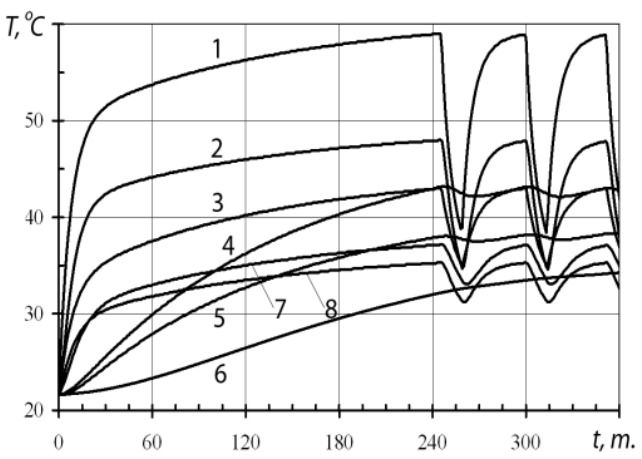

b)

Fig. 4. Changing of element temperature in the absence (a) and in the presence (b) of thermal control: 1 - NEST; 2 - inner wall surface in NEST zone; 3 - air; 4 - block; 5 - wall in block zone; 6 - bottom outside block; 7 - walls outside NEST; 8 - cover.

After the verification of the model, the simulation was performed for more complex variant of the container (see the schematic in Fig. 1), for which a system of 22 differential heat balance equations was formed. Initial data in the form of characteristics of NESTs, instrumentation blocks and materials to be applied when making the container were used (symbols of electric heaters and blocks are indicated in Fig. 1 and in the text). For NESTs, the following heat release power was adopted: $230 \mathrm{~W}$ (NEST 1), $135 \mathrm{~W}$ (NEST 2, 3), 180 W (NEST 4). For instrumentation blocks, weight as well as heat release power were taken into account (respectively): $52 \mathrm{~kg}, 90 \mathrm{~W}$ (block I); $15 \mathrm{~kg}, 115 \mathrm{~W}$ (block II); $30 \mathrm{~kg}, 0 \mathrm{~W}$ (block III); $25 \mathrm{~kg}, 0 \mathrm{~W}$ (block IV). The simulation of temperature fields of all elements of the container at ambient temperatures corresponding to winter and summer period was performed.

For winter period conditions, six variants of ambient temperature (from $-50{ }^{\circ} \mathrm{C}$ to $0{ }^{\circ} \mathrm{C}$ ) were considered. In this period, the temperature inside the container was maintained over 
the range of $+10{ }^{\circ} \mathrm{C} \ldots+25{ }^{\circ} \mathrm{C}$ that were provided with the NEST operation. Fig. 5 shows, as an example, temperature values for several elements.

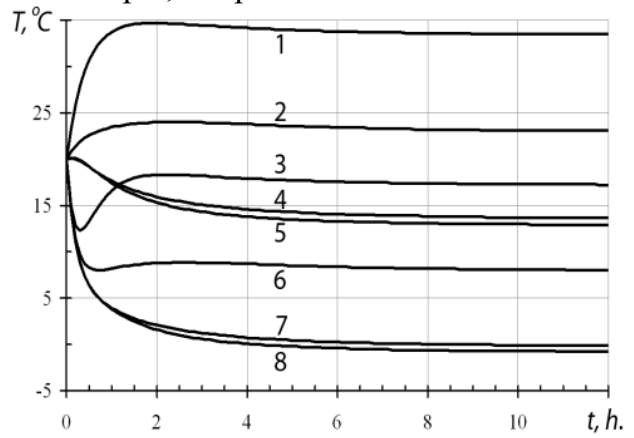

a)

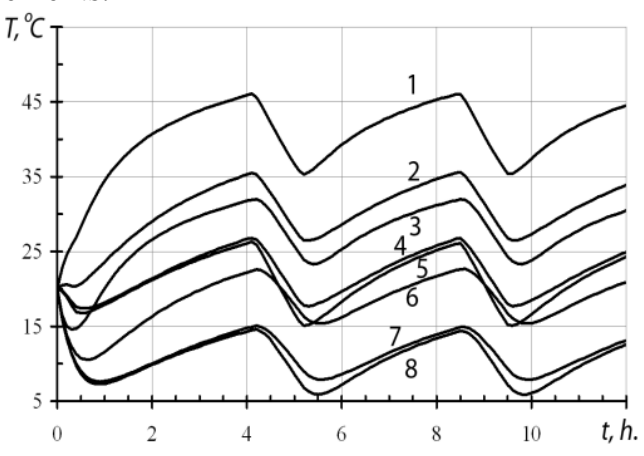

b)

Fig. 5. Changing of block temperatures (curves 1, 2, 4, 5) and wall surfaces $(3,6,7,8)$ in block zone (see Fig. 1): II $(1,3)$; I $(2,6)$; III $(4,7)$; IV $(5,8)$ at $T_{c}=-50{ }^{\circ} \mathrm{C}(\mathrm{a})$ and $-30^{\circ} \mathrm{C}(\mathrm{b})$.

It is ascertained that at ambient temperatures of $-50{ }^{\circ} \mathrm{C}$ and $-40{ }^{\circ} \mathrm{C}$ NESTs should operate permanently to maintain the scheduled temperature mode inside the container and at ambient temperature of $-30{ }^{\circ} \mathrm{C}$ the heaters can be cyclically switched out.

While analyzing the container work in summer period, the contribution of solar radiation to heating of vertical walls and a cover were taken into account. It was assumed that the air temperature inside the container is given over the range of $+25{ }^{\circ} \mathrm{C} \ldots+35^{\circ} \mathrm{C}$. The use of an air-conditioner (power of $400 \mathrm{~W}$ ) and a fun (volume air consumption of 0.07 $\mathrm{m}^{3} / \mathrm{s}$ ) at night was provided for. It was proposed that turn-on and turn-out of the airconditioner occurs under thermal control, and the fun operates only during the hours of darkness. The following variants of operating the container were modeled: without use of air-conditioner and fun; without use of air-conditioner but with operating fun; with use of air-conditioner but with switched-off fun (an example in Fig. 6).

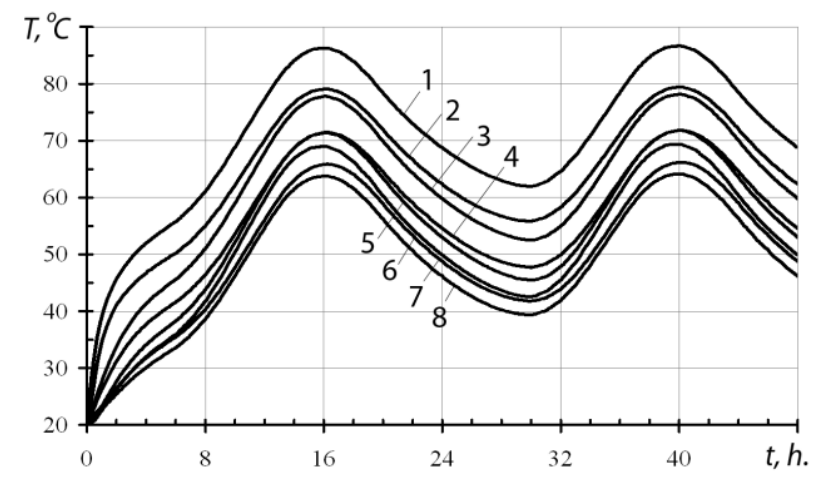

Fig. 6. Changing of temperatures of blocks and wall surfaces in block installation zone with switchedoff air-conditioner and switched-on fun (symbols of curves correspond to Fig. 5).

The obtained results, for example, show that the fun power at night is not sufficient for the stable operation of instrumentation blocks. It is shown that in this case it is expedient to use the air-conditioner that operates continuously. The simulation using developed software programs allows large volume of information important for practice to be obtained. It is possible, in particular, to perform the optimization of NEST number and power that provide a scheduled temperature level depending on the number and arrangement of 
instrumentation blocks. One may also choice rational materials and thicknesses of heat insulation of enclosing structures of containers and other PC products.

\section{Conclusion}

Thus, the developed method is an efficient instrument of simulating heat transfer processes in various PC products and can be considered as an alternative for commercial software complexes.

\section{References}

1. V.P. Gorelov, Low-temperature composite heaters in industry and private life (2016)

2. S.V. Baranov, O.N. Budadin, M.N. Slitkov, Russ. J. Nondestruct. 44, 2 (2008)

3. E.M. Sparrow, R.D. Cess. Radiation Heat Transfer (Hemispere Publishing Corporation, USA, 1978) 